\title{
Lengua Inglesa I. Talleres de dicción: pronunciación y voz pasiva
}

\author{
Yolanda Morató AgRofojo \\ Departamento de Filología \\ Inglesa (Lengua Inglesa) \\ Universidad de Sevilla \\ ymorato@us.es \\ ORCID: https://orcid.org/0000-0001-6540-6042 \\ D.0.1.: http://dx.doi.org/10.12795/JDU.2018.i01.94 \\ Pp.: 1676-1691
}

\section{Resumen}

Los distintos métodos y enfoques en la enseñanza de lenguas extranjeras han propiciado una mayor heterogeneidad, si cabe, en el aula. El objetivo de esta comunicación es conseguir que el nivel con el que parten los alumnos del primer año del grado en Estudios Ingleses alcance los estándares establecidos en el programa de la asignatura. Una vez realizado a principios del cuatrimestre un cuestionario inicial, en el que se les pide información sobre su aproximación al estudio de la lengua, se confirma que los alumnos desconocen ciertos aspectos relacionados con la dicción (oral y escrita) que son fundamentales para el desarrollo de su competencia comunicativa. Para ello, se plantean unos talleres basados en un ciclo de mejora docente (CMD) de la Universidad de Sevilla, en los que practicarán aspectos como la lectura de la transcripción de palabras en el diccionario y el uso apropiado de la voz pasiva. 
Palabras clave: Lengua Inglesa I, Estudios Ingleses, Docencia universitaria, experimentación docente universitaria, talleres de dicción

\section{Descripción del contexto de la experiencia docente}

Con sus 12 créditos, Lengua Inglesa I es una asignatura anual de primer curso del grado en Estudios Ingleses. El perfil de los alumnos matriculados en la asignatura es diverso, pero todos comparten unas determinadas características: han aprobado las pruebas de acceso a la Universidad $y$, por tanto, han hecho un examen con contenido lingüístico cuyo nivel de inglés se encuentra entre un B1 y un B2, es decir, ahora deberían demostrar una competencia lingüistica intermedia o intermedia alta. También es reseñable el hecho de que, al ser formación básica, se han tenido que matricular en la asignatura de manera obligatoria, aunque en torno al $15 \%$ ha entregado un certificado que acredita que ya cuentan con el nivel B2 o superior. Esta asignatura se imparte en 3 clases semanales de 80 minutos por sesión y asiste una media de 65 alumnos. Se imparte en un aula de informática de espacio reducido para el número de matriculados. Algunas personas se tienen que sentar en sillas supletorias, pues no hay ordenadores suficientes para cubrir la demanda.

\section{Diseño previo del Ciclo de Mejora Docente (CMD)}

Este CMD tiene lugar durante el segundo cuarto de la asignatura. Se tratan en él los temas que van desde la unidad 3B hasta la 5D (de un total de 10). Por ello, y con el objetivo de rentabilizar las unidades correspondientes a los 3 créditos de este CMD, se proponen talleres orientados a mejorar la dicción mediante actividades prácticas. El siguiente mapa de contenido representa la organización de los aspectos relacionados con la voz pasiva: 


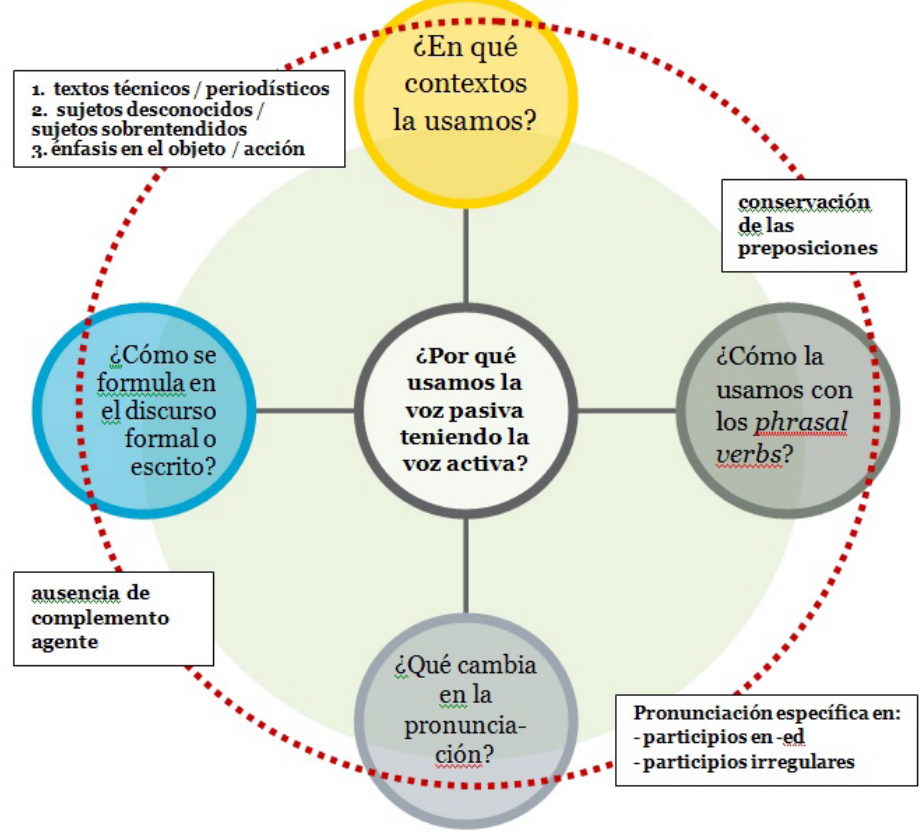

Colores de los círculos:

Act./Val.: fomentar un pensamiento basado en la aceptación de las diferencias entre lenguas y sus convenciones Conceptuales: utilizar la pasiva en contextos apropiados

Conceptuales.: producir inversiones en el discurso escrito

Datos: identificar patrones con phrasal verbs

Procedimentales Intelectuales: discriminar sonidos consonánticos finales

Figura 1. Mapa de contenido para la voz pasiva

En el mapa, los círculos incluyen preguntas generadoras de debate que coinciden con las preguntas planteadas en el cuestionario al inicio del CMD. Los elementos que se presentan dentro de los cuadrados se corresponden con el contenido de la unidad. En lugar de estudiar la voz pasiva desde una perspectiva estructuralista o basada en el método gramática-traducción, se presentan distintos aspectos que suelen plantear problemas entre los estudiantes.

Jornadas de Formación e Innovación Docente del Profesorado | № 1 (2018)

Esta obra se distribuye con la licencia Creative Commons Reconocimiento-NoComercial-SinObraDerivada Internacional (CC BY-NC-ND 4.0.) 
Modelo metodológico posible y secuencia de actividades diseñadas

El modelo metodológico que se plantea dentro de la estrategia docente propuesta en esta comunicación se resume en el esquema que ilustra la figura 2 :

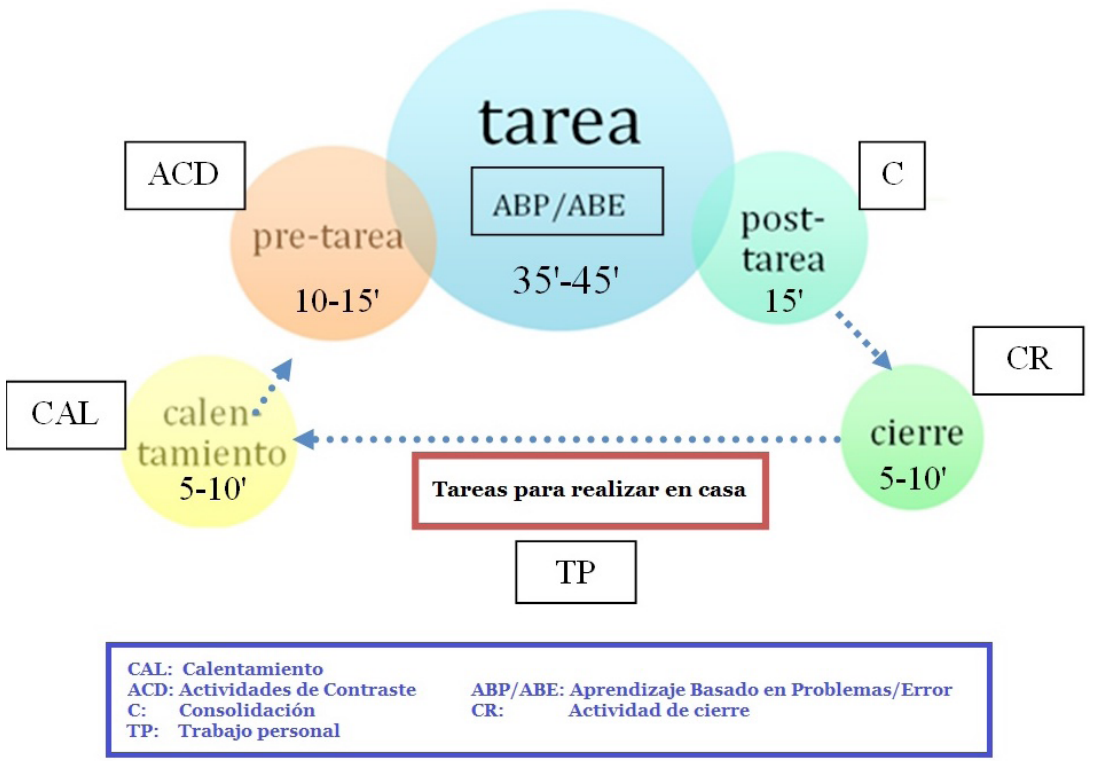

Figura 2. Modelo metodológico de las sesiones

En las siguientes tablas se muestran las actividades que se llevaron a cabo durante las cinco sesiones diseñadas para el segundo ciclo de mejora.

Jornadas de Formación e Innovación Docente del Profesorado | № 1 (2018) (i) Esta obra se distribuye con la licencia Creative Commons Reconocimiento-NoComercial-SinObraDerivada Internacional (CC BY-NC-ND 4.0.) 


\section{Tabla 1.}

Secuencia de actividades del ciclo de mejora

\begin{tabular}{|l|l|l|}
\hline \multicolumn{1}{|c|}{$\begin{array}{c}\text { Tipo de } \\
\text { actividad }\end{array}$} & \multicolumn{1}{|c|}{$\begin{array}{c}\text { Descripción de las actividades de la } \\
\text { sesión }\end{array}$} & Temporalización \\
\hline Calentamiento & $\begin{array}{l}\text { Ideas sobre los contenidos tratados } \\
\text { en la última sesión }\end{array}$ & $10-15^{\prime}$ \\
\hline $\begin{array}{l}\text { Actividad } \\
\text { de contraste }\end{array}$ & $\begin{array}{l}\text { Cuestionario en inglés siguiendo el } \\
\text { diseño y orientados a las escaleras } \\
\text { de aprendizaje en Porlán (2017) }\end{array}$ & $10-15^{\prime}$ \\
\hline ABE & $\begin{array}{l}\text { 1. Dictado del poema “One Art", de } \\
\text { Elizabeth Bishop. } \\
\text { 2. Corrección tras tres repeticiones } \\
\text { 3. Deciden qué palabras son las más } \\
\text { complicadas y por qué } \\
\text { 4. Consultan en sus ordenado- } \\
\text { res los significados y la transcrip- } \\
\text { ción fonética de los términos que } \\
\text { desconozcan }\end{array}$ & 40' \\
\hline C & $\begin{array}{l}\text { Discusión de errores involuntarios } \\
\text { en parejas como: } \\
\text { loose - lose, practice - practise }\end{array}$ & 10 ' \\
\hline CR & $\begin{array}{l}\text { ¿Escribimos envelop o envelope? } \\
\text { ¿Address o adress? (La influencia del } \\
\text { francés, idioma que también estu- } \\
\text { dian nuestros alumnos, les lleva a } \\
\text { confusiones). }\end{array}$ & 5' \\
\hline
\end{tabular}

\begin{tabular}{|l|l|l|}
\hline \multicolumn{1}{|c|}{$\begin{array}{c}\text { Tipo de } \\
\text { actividad }\end{array}$} & $\begin{array}{l}\text { Descripción de las actividades de la se- } \\
\text { sión 2 }\end{array}$ & Temporalización \\
\hline Calentamiento & $\begin{array}{l}\text { Presentación en clave de humor de un } \\
\text { sonido clave de la lengua inglesa no } \\
\text { presente en la nuestra: la schwa. }\end{array}$ & $5^{\prime}$ \\
\hline $\begin{array}{l}\text { Actividad de } \\
\text { contraste }\end{array}$ & $\begin{array}{l}\text { Lo visible y lo invisible: Debate en } \\
\text { grupo sobre letras que se escriben en } \\
\text { inglés y no se pronuncian. }\end{array}$ & $15^{\prime}$ \\
\hline ABP & $\begin{array}{l}\text { Pares mínimos l: vocales } \\
\text { ¿Cuáles son los principales sonidos } \\
\text { que ocasionan problemas a los ha- } \\
\text { blantes no nativos de inglés? Este pro- } \\
\text { blema consistirá en varios pasos y se } \\
\text { llevará a cabo en tres sesiones. La pri- } \\
\text { mera, esta, tratará de las vocales. De- } \\
\text { batirán y veremos soluciones en vídeos } \\
\text { dedicados a la fonética }\end{array}$ & \\
\hline
\end{tabular}




\begin{tabular}{|l|l|l|}
\hline Tarea para casa & $\begin{array}{l}\text { Breve lectura relacionada con el con- } \\
\text { tenido de la unidad "What is a Schwa? } \\
\text { Helping Students Read and Spell the } \\
\text { Schwa Sound" }\end{array}$ & 20 \\
\hline
\end{tabular}

\begin{tabular}{|l|l|l|}
\hline \multicolumn{1}{|c|}{$\begin{array}{c}\text { Tipo de } \\
\text { actividad }\end{array}$} & \multicolumn{1}{|c|}{ Descripción de las actividades de la } \\
sesión 3 & \multicolumn{1}{|c|}{ Temporalización } \\
\hline Calentamiento & $\begin{array}{l}\text { En un vídeo, varios hablantes discuten } \\
\text { a la entrada de la British Library la me- } \\
\text { jor manera de pronunciar varias pala- } \\
\text { bras. Se propone un debate en grupos } \\
\text { sobre las palabras que mayores pro- } \\
\text { blemas generan. Los alumnos elaboran } \\
\text { una lista y se intercambia. }\end{array}$ & \\
\hline ABP & $\begin{array}{l}\text { Pares mínimos II.1: consonantes } \\
\text { ¿Cuáles son los principales sonidos } \\
\text { que ocasionan problemas a los ha- } \\
\text { blantes no nativos de inglés? 2o sesión } \\
\text { sobre las consonantes. Debatirán y ve- } \\
\text { remos soluciones en vídeos dedicados } \\
\text { a la fonética. }\end{array}$ & 45' \\
\hline C & $\begin{array}{l}\text { Test con preguntas autocorregibles en } \\
\text { Kahoot! El objetivo es reforzar la prác- } \\
\text { tica de palabras que contienen conso- } \\
\text { nantes mudas. }\end{array}$ & 10' \\
\hline Cierre & $\begin{array}{l}\text { Elaboran una lista en su cuaderno con } \\
\text { las palabras que han fijado correcta- } \\
\text { mente después de la práctica (a ser } \\
\text { posible, transcripción). }\end{array}$ & 5' \\
\hline Tarea para & $\begin{array}{l}\text { Ejercicios prácticos (de naturaleza pro- } \\
\text { cedimental) de la BBC: http://www.bbc. } \\
\text { co.uk/worldservice/learningenglish/ } \\
\text { grammar/pron/features/schwa/ }\end{array}$ & \\
\hline
\end{tabular}

\begin{tabular}{|c|l|l|}
\hline $\begin{array}{c}\text { Tipo de } \\
\text { actividad }\end{array}$ & \multicolumn{1}{c|}{$\begin{array}{c}\text { Descripción de las actividades de la } \\
\text { sesión 4 }\end{array}$} & Temporalización \\
\hline Calentamiento & $\begin{array}{l}\text { En parejas discuten cuántas veces } \\
\text { aparece la schwa en la siguiente frase: } \\
\text { "To survive the cold weather you have } \\
\text { to make thorough preparations" }\end{array}$ & $10-15^{\prime}$ \\
\hline
\end{tabular}

Jornadas de Formación e Innovación Docente del Profesorado I № 1 (2018) Esta obra se distribuye con la licencia Creative Commons 


\begin{tabular}{|l|l|l|}
\hline ABP & $\begin{array}{l}\text { Pares mínimos II.2: consonantes } \\
\text { ¿Cuáles son los principales sonidos } \\
\text { que ocasionan problemas a los ha- } \\
\text { blantes no nativos de inglés? 3á sesión } \\
\text { sobre las consonantes. Debatirán y ve- } \\
\text { remos soluciones en vídeos dedicados } \\
\text { a la fonética. }\end{array}$ & 45' \\
\hline C & $\begin{array}{l}\text { Reciben un texto que editarán, corri- } \\
\text { giendo aquellos aspectos lingǘsticos } \\
\text { que no sean correctos en inglés. }\end{array}$ & 10' \\
\hline Cierre & $\begin{array}{l}\text { Elaboran una lista en su cuaderno } \\
\text { con las palabras que han fijado des- } \\
\text { pués de la práctica (a ser posible, con } \\
\text { transcripción) }\end{array}$ & 5-10' \\
\hline $\begin{array}{l}\text { Tarea para } \\
\text { casa }\end{array}$ & $\begin{array}{l}\text { Comprueban en casa si han corre- } \\
\text { gido bien los errores y escriben un in- } \\
\text { forme sobre aquellos que no se han } \\
\text { detectado. }\end{array}$ & 30 \\
\hline
\end{tabular}

\begin{tabular}{|l|l|l|}
\hline \multicolumn{1}{|c|}{$\begin{array}{c}\text { Tipo de } \\
\text { actividad }\end{array}$} & \multicolumn{1}{|c|}{$\begin{array}{c}\text { Descripción de las actividades de la } \\
\text { sesión 5 }\end{array}$} & Temporalización \\
\hline Calentamiento & $\begin{array}{l}\text { Mapa conceptual en grupo. Escribo } \\
\text { una palabra en la pizarra y les pido } \\
\text { otras relacionadas, que se van colo- } \\
\text { cando según las relaciones que se es- } \\
\text { tablezcan entre ellas. }\end{array}$ & $10-15^{\prime}$ \\
\hline ABE & $\begin{array}{l}\text { Dictado del poema “Prufrock", del pre- } \\
\text { mio Nobel T. S. Eliot. } \\
\text { Pasos como en la sesión 1. }\end{array}$ & 45' \\
\hline C & $\begin{array}{l}\text { Dramatic reading: leen en alto un frag- } \\
\text { mento del guion de una serie o pelí- } \\
\text { cula, para practicar la pronunciación y } \\
\text { la entonación. }\end{array}$ & \\
\hline CR & $\begin{array}{l}\text { If you weren't here, where would you } \\
\text { like to be?: Actividad en la que se fo- } \\
\text { menta la respuesta a una situación } \\
\text { hipotética, con la que practican las es- } \\
\text { tructuras condicionales }\end{array}$ & 5' \\
\hline
\end{tabular}

\section{Cuestionario previo}

A continuación se presenta el cuestionario que se distribuyó entre los estudiantes en inglés (la versión en español se puede consultar en el mapa de contenidos): 
1. Why do we use the passive voice in English (it was broken) instead of the active voice (I broke it)?

2. Write a passive sentence which includes the phrasal verb to break into.

3. Translate into English using a passive structure: «A ella se le dijo que trajera el libro».

4. Think of any passive sentence in English. What is the main element that we should take care of in pronunciation?

5. In what contexts do we use the passive voice?

\section{Aplicación del CMD}

\section{Sesión 1}

Reparto el cuestionario en papel que he diseñado en nuestro curso y les pido que lo completen con total sinceridad. Puedo ver que algunos no entienden bien las preguntas, porque no están acostumbrados a responder a cuestiones «híbridas» en una asignatura instrumental; es decir, no son preguntas estrictamente lingüísticas y algunas tampoco son del todo prácticas. Cuando terminan empezamos una actividad auditiva: el dictado del poema «One Art», de Elizabeth Bishop. Cuando escuchan el poema entero por primera vez, se oyen resoplidos en el aula. He elegido un poema con una lectura muy lenta. La falta de familiaridad con este tipo de ejercicio (que ha sido muy criticado por algunos pedagogos) y el propio género poético les desconcierta, pero están en un grado en el que van a trabajar con distintas composiciones poéticas y no cuentan con demasiada formación al respecto. Además, los dictados y las lecturas reciben el principal peso de la evaluación en la prueba de enero. Una vez que hemos completado todos los versos, repitiéndolos dos y hasta tres veces, comprobamos el resultado con subtítulos en la versión en pantalla. Como parecen agotados, para cambiar el 
ritmo, les pido que trabajen en parejas unos minutos y decidan qué palabras son las más complicadas y por qué.

Cuando se acaba el tiempo para el debate, les pido que digan en voz alta los resultados a los que han llegado. No son los que yo esperaba (mi actividad de consolidación estaba orientada al repaso de determinados errores frecuentes), pero los anoto en la pizarra y ellos en sus cuadernos. A continuación, les planteo las dificultades que había identificado (grafías y categorías gramaticales). Algunos gritan inesperadamente que ellos han tenido mal esas palabras. Para mis próximas sugerencias de debate, reformularé mis instrucciones. Le pediré que de los errores que han cometido, seleccionen tres, y que expliquen por qué creen que les ha salido mal y qué pueden hacer para solucionarlo. Las palabras que he preparado para la última reflexión les ayudan a darse cuenta de que, en el fondo, nuestra lengua materna nos hace parecernos tanto que incluso cometemos los mismos errores. Esto, les digo, es una buena noticia, porque nos permite trabajar de manera consciente en la mejora de nuestras destrezas, identificando nuestros puntos débiles y practicando mucho para eliminarlos, como hacen los deportistas. Lo que ha funcionando mejor ha sido la actividad centrada en la corrección y la posterior reflexión sobre los errores que han cometido. Por el camino, hemos repasado una antigua regla ortográfica sobre la duplicación de consonantes que muchos desconocían o habían aprendido mal. Me gusta trabajar sobre el error en clase porque genera un clima de mayor confianza y, además, la autocorrección o la corrección por parte de otros compañeros (peer review) es una herramienta de mejora muy beneficiosa.

Sesión 2

Como ya sé por los cuestionarios, mis alumnos no están familiarizados con la fonética en lengua inglesa, por lo que uso las imágenes humorísticas para propiciar el 
debate e intentar que asocien el sonido con la imagen (así será una experiencia con mayor posibilidad de ser memorable que un simple símbolo). Aprovecho esta circunstancia para unir la imagen que he utilizado para el calentamiento con la actividad de contraste: sondear qué saben mis alumnos sobre las letras que no se pronuncian en inglés y ver qué pronunciaciones erróneas han fosilizado.
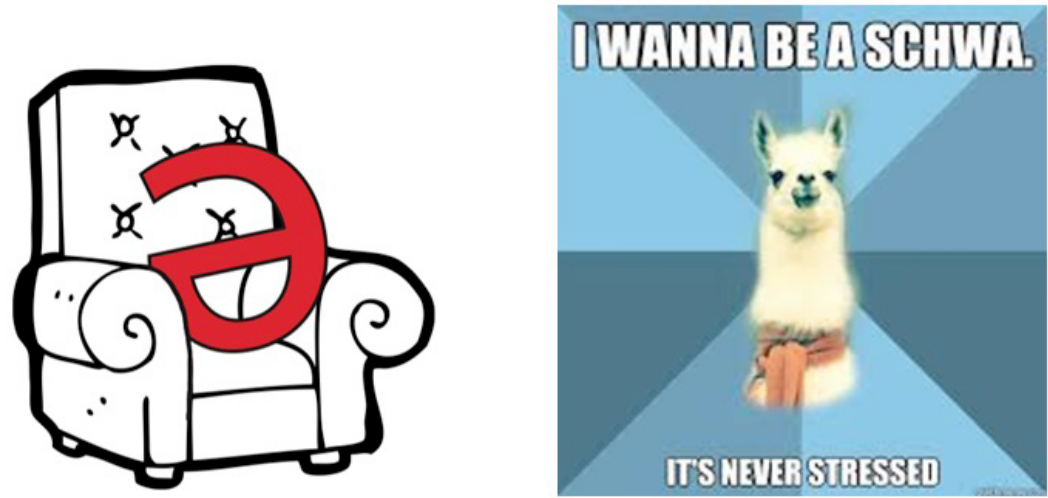

Figura 3. Representaciones gráfico-humorísticas de la schwa (https://goo.gl/ wBdiZS y https://goo.gl/udYFV3)

He titulado la actividad de contraste "Lo visible y lo invisible», porque muchas de las cosas que necesitamos mejorar en nuestra práctica profesional residen fundamentalmente en el espacio lingüístico de lo invisible, o, al menos, en aquellas cosas que no son evidentes a primera vista, en los aspectos que se nos escapan por las prisas y el descuido en nuestra vida cotidiana. Les doy una lista de palabras y les pido que localicen las consonantes que no se pronuncian. Son: climbing, bomb, plomber, famous, debt y doubt. En todas ellas la «b» es muda.

A partir de este punto la clase resulta fluida y el hilo conector de las actividades queda bastante claro. Para mejorar la pronunciación de manera consciente, el «problema» para esta sesión consiste en buscar los sonidos que no tenemos en nuestras lenguas maternas (hay varios alumnos 
extranjeros en el aula). Para ello, comenzamos por las vocales. Empezamos por escribir las cinco vocales en una columna vertical y dividimos en dos el eje horizontal: vocales cortas y largas. Lo hacen en parejas o grupos (según la agrupación de ordenadores) y entre todos ellos llegan a localizar 10 de las 15 que les propongo. No saben escribir todas ellas, así que dividimos el intercambio de información por vocales y proyecto tres vídeos de la BBC con ejemplos específicos e instrucciones sobre cómo articularlas. Los alumnos participan en las tareas de producción sin que se les tenga que pedir de manera explícita, por lo que se obtienen prácticas muy colaborativas.

\section{Sesiones 3 y 4}

Como en las sesiones anteriores, completamos las actividades de pronunciación tal como aparecen en el diseño y las terminan todas en el tiempo estipulado. Ahora ya empiezan a hacer consultas sobre «problemas» análogos a aquellos que se presentan en las actividades, por lo que puedo ver que están muy involucrados en las tareas. Son muy participativos y, aunque manejar un grupo de casi setenta alumnos es complicado, el clima de trabajo se restablece con bastante facilidad. Al final de las clases varias alumnas han solicitado más ejercicios como los que hemos hecho en clase.

\section{Sesión 5}

Hemos acabado las sesiones dedicadas a la práctica de lectura fonética, cuyo principal objetivo no era ofrecerles la formación en profundidad que recibirán en la asignatura obligatoria de 20 «Fonética y Fonología del Inglés», sino que puedan leer la transcripción de las palabras en los distintos diccionarios que manejan y empezamos a conectar ideas. He cambiado el dictado que había planificado por otro tipo de grabación, para no repetir el mismo género. En este caso, he elegido el mensaje de un contestador 
automático, pues es un tipo de documento sonoro real, al que se enfrentarán tarde o temprano. El segmento con el que trabajamos (https://www.youtube.com/watch?v=8Ccn07kMXmw) es muy útil tanto para el dictado como para su posterior análisis.

La actividad de dramatic reading consiste en leer un texto interpretándolo, para practicar la entonación, el ritmo y la pronunciación, entre otras cosas. En sus cuestionarios de principios de curso algunos me dijeron que les gustaban las series, por lo que les doy la página 16 del pdf (https://filmschoolrejects.com/wp-content/ uploads/2017/05/Screenplay-Breaking Bad-Pilot.pdf), que corresponde al episodio piloto de la serie Breaking Bad. Lo practican en parejas y parecen muy animados. Termino con el resto de la planificación.

Estas sesiones han sido muy satisfactorias. En poco tiempo he podido conectar con los alumnos y el ritmo de trabajo ha sido el que había programado, algo que no siempre sucede. Es un buen grupo que siempre está dispuesto a participar en todas las tareas que les propongo, incluso cuando es la primera vez que las hacen. Ahora estoy inmersa en el proceso de adaptar el resto de los créditos que tengo al modelo que ya he puesto en marcha.

\section{Evaluación del CMD}

Debido a los plazos con los que contamos, se han examinado 25 cuestionarios seleccionados al azar. Aunque las preguntas se formularon en inglés, se ofrece aquí una traducción de las dos primeras preguntas para facilitar la lectura de aquellos compañeros que no leen en inglés. Uno de los retos ha sido elaborar una redacción que hiciera reflexionar sobre la estructura profunda de la lengua en todas sus dimensiones, pero que también contuviese aspectos prácticos. Para su análisis y representación gráfica mediante escaleras de aprendizaje he seleccionado la primera pregunta (de cinco), orientada a recabar 
información de tipo conceptual y la segunda, en la que se pide una aplicación práctica del tema y se solicita una respuesta de contenido procedimental.

Cuestión 1. ¿Por qué usamos la voz pasiva en inglés en vez de la activa?

A. Usos formales en textos escritos

B. Para no reconocer la responsabilidad del acto/hacerlo más impersonal

C. No dar importancia al sujeto/énfasis del sujeto agente/de las acciones

D. No sé

E. Interpretaciones incorrectas o que no responden a la pregunta: algo acaba de pasar/acción en el pasado/necesitamos un sujeto paciente/el sujeto es it/ énfasis en el verbo/la pasiva es más correcta

A $12 \% \quad 68 \%$

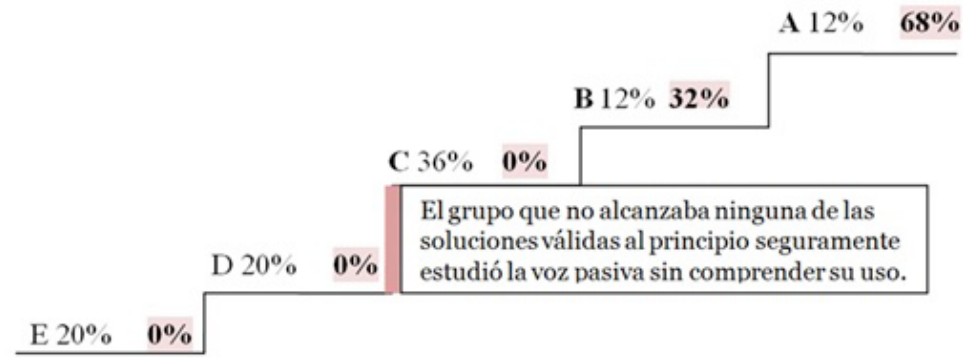

Cuestión 2. Escribir una oración pasiva con el plurasal verb to break into

A. Pasiva correcta (sin sujeto agente, sobreentendido por el propio verbo)

B. Pasiva con sujeto agente redundante

C. Pasiva sin el verbo conjugado/significado de break into incorrecto

D. No sé

E. Pasiva incorrecta

F. No se puede/es incorrecto

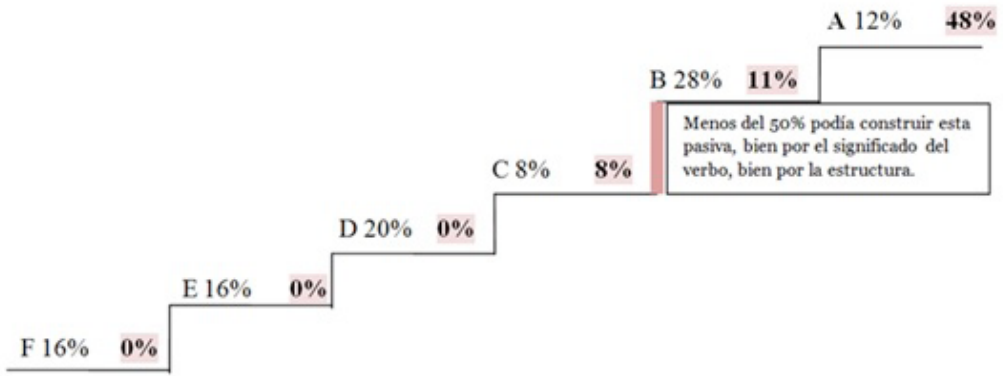

Figura 4. Escaleras de aprendizaje con los porcentajes iniciales (en blanco) y finales (en rosa) 
Tabla 2. Cuadrante analítico de las dos preguntas al principio (INI) y al final (FIN)

\begin{tabular}{|c|c|c|c|c|c|c|c|c|c|c|c|c|c|}
\hline \multirow{2}{*}{ Alumno } & \multicolumn{2}{|c|}{ Pregunta 1} & \multirow{2}{*}{$\begin{array}{c}\text { Cambio } \\
=/+/-\end{array}$} & \multicolumn{2}{|c|}{ Pregunta 2} & \multirow{2}{*}{$\begin{array}{c}\text { Cambio } \\
=/+/-\end{array}$} & \multirow{2}{*}{ Alumno } & \multicolumn{2}{|c|}{ Pregunta 1} & \multirow{2}{*}{$\begin{array}{c}\text { Cambio } \\
=1+1-\end{array}$} & \multicolumn{2}{|c|}{ Pregunta 2} & \multirow{2}{*}{$\begin{array}{c}\text { Cambio } \\
=1+1-\end{array}$} \\
\hline & INI & FIN & & INI & FIN & & & INI & FIN & & INI & FIN & \\
\hline 1 & A & A & $=$ & $\mathrm{D}$ & B & +2 & 14 & $\mathrm{C}$ & A & +2 & $\mathrm{~F}$ & B & +4 \\
\hline 2 & A & A & $=$ & $\mathrm{E}$ & B & +3 & 15 & B & A & +1 & B & A & +1 \\
\hline 3 & A & A & $=$ & $\mathrm{E}$ & B & +3 & 16 & $\mathrm{E}$ & A & +4 & D & B & +2 \\
\hline 4 & E & B & +3 & $\mathrm{~F}$ & B & +4 & 17 & $\mathrm{D}$ & A & +3 & B & A & +1 \\
\hline 5 & $\mathrm{C}$ & A & +2 & $\mathrm{C}$ & B & +2 & 18 & $\mathrm{D}$ & B & +2 & B & A & +1 \\
\hline 6 & $\mathrm{C}$ & A & +2 & D & A & +3 & 19 & B & A & +1 & A & A & $=$ \\
\hline 7 & $\mathrm{C}$ & A & +2 & $\mathrm{~F}$ & B & +4 & 20 & $\mathrm{E}$ & B & +3 & B & A & +1 \\
\hline 8 & $\mathrm{D}$ & A & +3 & $\mathrm{D}$ & B & +2 & 21 & $\mathrm{D}$ & B & +2 & B & A & +1 \\
\hline 9 & B & A & +1 & A & A & $=$ & 22 & $\mathrm{C}$ & A & +2 & B & A & +1 \\
\hline 10 & $\mathrm{E}$ & A & +4 & $\mathrm{E}$ & B & +2 & 23 & $\mathrm{C}$ & A & +2 & A & A & $=$ \\
\hline 11 & $\mathrm{C}$ & B & +2 & $\mathrm{~F}$ & $\mathrm{C}$ & +3 & 24 & $\mathrm{C}$ & A & +2 & B & A & +1 \\
\hline 12 & $\mathrm{D}$ & B & +2 & $\mathrm{C}$ & B & +1 & 25 & $\mathrm{E}$ & B & +3 & $\mathrm{C}$ & A & +2 \\
\hline 13 & $\mathrm{C}$ & B & +1 & $\mathrm{D}$ & $\mathrm{C}$ & +2 & & & & & & & \\
\hline
\end{tabular}

Como puede observarse en la representación gráfica y en el cuadro analítico, los resultados de las actividades han sido excelentes, como se constata en las zonas sombreadas en rosa, que corresponden a los logros finales. Me gustaría ser precavida y volver a pasar el cuestionario dentro de dos meses, pues tengo la sensación de que tienen los conceptos muy recientes y es necesario dejar pasar tiempo para analizar si se han asentado. La evaluación es un elemento que siempre aparece al principio, cuando se presenta el programa, y al final, cuando los alumnos vuelven a preguntarse qué y cómo se va a evaluar en el examen. Sin embargo, la evaluación es, en realidad, todo lo que pasa entre ese primer día y los últimos. No entenderlo así es fruto de una mala concepción o planificación docente. Debo encontrar, por otra parte, el equilibrio para diseñar actividades de evaluación compatibles con la carga de trabajo que suponen 70 alumnos. Utilizaré tareas grupales para guiar aquellas actividades en las que ellos puedan gestionar los procesos de corrección y mejora, con el fin de que descubran en la práctica las implicaciones de los distintos criterios de evaluación.

Jornadas de Formación e Innovación Docente del Profesorado I № 1 (2018) Esta obra se distribuye con la licencia Creative Commons 
Tras haber evaluado el diseño y su aplicación, y gracias a la lectura de obras clave como las de Finkel (2000, trad. 2008) y Bain (2004, trad. 2007), me gustaría citar cinco pilares fundamentales para nuestra labor docente:

- Los alumnos son el centro del aprendizaje dentro y fuera del aula; y resulta esencial que puedan transferir el conocimiento que adquieren a otras situaciones en el seno de la sociedad

- La selección y el diseño meticuloso del contenido contribuye a la actualización continua de los saberes fundamentales de las disciplinas en las que somos especialistas

- El desarrollo de una metodología estructurada e integrada en la planificación favorece el andamiaje necesario para que los alumnos construyan el conocimiento por sí mismos

- La reflexión sistematizada sobre la práctica docente mediante distintas herramientas nos ayuda a mantener un equilibrio en nuestra labor

- La evaluación como una práctica continua que promocione el pensamiento crítico debe apoyarse en diversos instrumentos y estrategias, entre otras: preguntas encadenadas, hipótesis y problemas, portafolios, proyectos o retos

\section{Conclusiones}

Durante este CMD he dedicado largos periodos de reflexión los tipos de contenidos de la lengua inglesa y su posible jerarquización. A pesar de lo duro que me resultó conceptualizar y problematizar la lengua, no me arrepiento del tiempo invertido; ha sido un proceso de aprendizaje muy interesante. Con el paso de los años, los docentes de lenguas adquirimos una especie de banco de errores mental que nos ayuda a anticipar aquellos espacios lingüísticos en los que un buen número de estudiantes dudará o flaqueará. Por tanto, el problema no residía en conocer 
más a fondo la lengua, sino en encontrar maneras de sacar ese fondo a la superficie más allá de los libros de texto, el material audiovisual (que tan desarrollado está en estos momentos) o las explicaciones basadas en el modelo transmisivo. He querido representarlos de manera visual y encadenar las preguntas pertinentes para que esos espacios lingüísticos dejaran de ser territorios hostiles o, simplemente, lugares insospechados para el error. Para ello, he acudido a los principios de Ken Bain, buscando establecer rutinas que, mediante el uso de preguntas, desarrollen el pensamiento crítico y el razonamiento disciplinar en el aprendizaje del inglés.

\section{Bibliografia}

Bain, K. (2007). Lo que hacen los mejores profesores universitarios. Valencia: Universidad de Valencia.

Fnkel, D. (2008). Dar clase con la boca cerrada. Valencia: Universidad de Valencia.

Porlán, R. (2017). Enseñanza universitaria. Cómo mejorarla. Madrid: Morata.

Jornadas de Formación e Innovación Docente del Profesorado | № 1 (2018) Esta obra se distribuye con la licencia Creative Commons 